\title{
ESTRATEGIAS PARA EL MODELADO Y EL ANÁLISIS SÍSMICO DE ESTRUCTURAS HISTÓRICAS
}

\author{
Fernando Peña Mondragón ${ }^{(1)}$
}

\begin{abstract}
RESUMEN
Con el fin de evitar mutilar la componente histórica y salvaguardar los valores intrínsecos de una estructura histórica durante una intervención, es necesario que el proyecto estructural esté basado en el "entendimiento total" del comportamiento estructural del inmueble. El comportamiento estructural de un edificio antiguo de mampostería difiere sustancialmente de una estructura moderna. Por lo que, los estudios analíticos de este tipo de estructuras requieren de procedimientos específicos. En este artículo se presenta una serie de estrategias para el modelado y el análisis del Patrimonio Arquitectónico. Así, se describe la estrategia y se presentan algunos ejemplos ilustrativos.
\end{abstract}

Palabras Clave: Estructuras históricas; análisis sísmico; estrategia integral de análisis complementarios; mampostería

\begin{abstract}
With the purpose of avoiding to mutilate the historical component and to safeguard the intrinsic values of a historical structure during an intervention, it is necessary that the structural project is based on the total understanding of the structural behavior of the building. The structural behavior of an old masonry building is completely different from a modern structure. Thus, the analytical studies of these structures require specific procedures. In this paper a strategy for modeling and analyzing historical masonry buildings is presented. The approach is drawn, as well as some examples are presented.
\end{abstract}

Key Words: Historical structures; seismic analysis; integrate approach of complementary analysis; masonry

\section{INTRODUCCIÓN}

Debido a que el daño estructural de un edificio histórico tiene consecuencias no sólo a nivel de costos o pérdidas humanas, sino también a nivel cultural y patrimonial, el estudio de la seguridad estructural de este tipo de construcciones es de vital importancia. De este modo es necesario que el proyecto estructural esté basado en la "comprensión total” del comportamiento estructural del inmueble, así como de las características de sus materiales. Así mismo, el diagnóstico y evaluación de la seguridad deben estar basados tanto en información histórica, como en criterios adecuados para la conservación de las edificaciones históricas. Estos criterios deben de reconocer explícitamente que la conservación del

Artículo recibido el 8 de octubre de 2010 y aprobado para su publicación el 20 de diciembre de 2010. Seaceptarán comentarios y/o discusiones hasta cinco meses después de su publicación

1 Instituto de Ingeniería, Universidad Nacional Autónoma de México. Edificio 2, Circuito Escolar, Ciudad Universitaria, 04510, México, DF. fpem@pumas.iingen.unam.mx 
Patrimonio Arquitectónico se debe centrar en tres puntos fundamentales: la seguridad física de las personas, la salvaguarda de los valores intrínsecos del inmueble y el uso actual y futuro que se le dé a la construcción.

Así mismo, el comportamiento estructural de un edificio antiguo de mampostería difiere sustancialmente de una estructura moderna. Esto se debe, principalmente, al tipo de estructuración y a los materiales usados. Por lo que, los estudios analíticos de este tipo de estructuras requieren de procedimientos específicos. Debido a esto, es necesario que el ingeniero conozca los materiales y técnicas constructivas usados en el pasado para que pueda hacer una buena descripción del comportamiento estructural del edificio histórico.

A pesar de que en la actualidad se cuenta con teorías y técnicas de análisis que permiten modelar numéricamente y con gran precisión el comportamiento de estructuras complejas, el análisis estructural de edificios históricos de mampostería continúa siendo un desafío debido a una serie de factores, principalmente a que la mampostería es un material compuesto que presenta elevados valores de resistencia a compresión y prácticamente no resiste tensiones lo que dificulta proponer modelos que reproduzcan adecuadamente este tipo de comportamiento (Orduña et al., 2007). Así mismo, surgen otra serie de factores que no son de índole numérica o matemática, pero que afectan la realización y análisis de los modelos numéricos. Como por ejemplo, la descripción geométrica y estructural del edificio, la identificación de los materiales, la variabilidad de la calidad y propiedades mecánicas de los materiales, entre otros.

Por otro lado, como el objetivo de una intervención en un edificio histórico es la salvaguarda de los valores intrínsecos del edificio (Peña y García Córdova, 2004) y no sólo de la seguridad estructural del inmueble, es necesario que el análisis estructural no esté basado únicamente en un análisis numérico, como se sobrentiende comúnmente en ingeniería; sino que esté referido a un análisis más completo que lleve a la comprensión total del comportamiento de la edificación. Es decir, que la evaluación de la seguridad estructural no debe estar basada únicamente en cálculos numéricos, sino también en las evidencias sobre el comportamiento del edificio ante eventos pasados.

Tomando en cuenta lo anterior, en este artículo se presenta una serie de estrategias para el modelado y el análisis de estructuras históricas, que en este trabajo se ha llamado: “Estrategia Integral de Análisis Complementarios”. Esta estrategia permite superar las dificultades inherentes al análisis de estructuras históricas a través de una serie de análisis que se complementan entre sí. Así, se describe la estrategia y se muestran algunos ejemplos ilustrativos.

\section{VALORES INTRÍNSECOS DE UNA ESTRUCTURA HISTÓRICA}

En estructuras modernas, los niveles mínimos de seguridad y servicio se encuentran especificados en los reglamentos y normas de construcción. Por ejemplo, el objetivo de las Normas Técnicas Complementarias para Diseño por Sismo del Reglamento del Distrito Federal es (NTC-S, 2004): “obtener una seguridad adecuada tal que, bajo el sismo máximo probable, no habrá fallas estructurales mayores ni pérdidas de vidas, aunque pueden presentarse daños que lleguen a afectar el funcionamiento del edificio y requerir reparaciones importantes”. Sin embargo, cuando se interviene una estructura histórica la seguridad adecuada que se busca va más allá de evitar fallas estructurales mayores y pérdidas de vidas. Se debe buscar, sobretodo, la salvaguarda de los “valores intrínsecos” del inmueble. Lo que requiere que se utilice un criterio específico para este tipo de estructuras. 
Cuando hablamos de los valores intrínsecos de una estructura histórica nos referimos a todo aquello que se podría perder, sea tangible o intangible, en caso de la desaparición del inmueble histórico. Grosso modo podemos clasificarlos en cinco valores:

- Arquitectura. Además del estilo arquitectónico, la distribución de espacios, usos y modificaciones a lo largo de su historia forman parte del valor cultural del edificio histórico.

- Arte. El valor artístico reside tanto en su arquitectura, como en todos los demás elementos que le proporcionan una identidad al edificio, como pueden ser: frescos y pinturas murales, elementos de adorno (cielos rasos, adornos en columnas y muros, pisos, etc.), bienes muebles conservados en el interior del edificio, etc.

- Económico. Los edificios y centros históricos son, muchas veces, la atracción principal de una ciudad, creando beneficios económicos directos e indirectos.

- Historia. El valor cultural histórico de un inmueble no se refiere únicamente a la época en que fue construido, sino también a todos los hechos de que ha sido testigo mudo. Como por ejemplo, ser la casa en donde vivió cierto personaje de la historia, lugar donde se redactó o firmó cierto acuerdo.

- Ingeniería. La concepción estructural de un edificio histórico es también parte de su valor cultural. Ésta muestra en forma tangible las antiguas técnicas de construcción y los materiales usados. Las estructuras de los monumentos constituyen sin duda alguna un documento histórico y un legado vivo de las habilidades de los antiguos constructores.

Lamentablemente, muchas veces el ingeniero no reconoce o toma en cuenta estos valores para proponer el proyecto de intervención. Otras veces, se reconocen sólo algunos de ellos (como arte, historia y arquitectura), pero la falta de información sobre las técnicas y materiales de construcción de épocas pasadas hacen que el valor ingeniería se desprecie y se pierda o mutile al proponer una rehabilitación con técnicas modernas y novedosas.

\section{PRINCIPIOS DE CONSERVACIÓN Y CRITERIOS PARA EL ANÁLISIS Y RESTAURACIÓN DE ESTRUCTURAS HISTÓRICAS}

El proceso de intervención de cualquier tipo de estructura debe incluir: la identificación del tipo de falla (levantamiento de daños); el diagnóstico de la seguridad estructural (si el daño es leve, moderado, severo), la propuesta de una o varias técnicas de intervención, y finalmente, se debe realizar una evaluación de la eficiencia del procedimiento de intervención, es decir, si es económica, técnicamente viable, funcional, etc. Así mismo, el proceso de intervención debe garantizar que se obtenga una seguridad adecuada.

La combinación del conocimiento científico y cultural con la experiencia es indispensable para la conservación y estudio del patrimonio arquitectónico. El objetivo final de cualquier estudio, investigación e intervención debe ser la salvaguarda de los valores intrínsecos del edificio en su totalidad. De este modo, la Ingeniería de la Conservación es el medio científico con el cual se logra este resultado (Lourenço, 2006). De este modo, Lourenço (2006) define una metodología para la intervención estructural de un edificio histórico, siguiendo los principios ISCARSAH de ICOMOS (2003), la que se puede dividir en cuatro aspectos principales:

- Adquisición de Datos

- Comportamiento Estructural

- Diagnóstico y Seguridad 


\section{- Medidas de Intervención}

La aplicación de esta metodología requiere un proceso iterativo entre la adquisición de datos, el estudio del comportamiento estructural, el diagnóstico y la evaluación de la seguridad de la estructura. Las medidas de intervención estarán basadas en el diagnóstico y la evaluación de la seguridad. Por lo tanto, el desarrollo incorrecto de estos pasos llevará a una conclusión errónea, que en algunos casos puede llegar a la destrucción total de los valores intrínsecos del inmueble. De este modo, el diagnóstico y evaluación de la seguridad deben estar basados en información histórica (Peña y García Córdova, 2004), así como en procedimientos cualitativos y cuantitativos. Los procedimientos cualitativos se basan en la observación directa del daño estructural y de la degradación de los materiales, así como de una investigación histórica. Los procedimientos cuantitativos se basan principalmente en pruebas realizadas a los materiales y a la estructura en general, monitoreo y análisis estructural.

Por otro lado, las medidas de intervención deben estar basadas en las causas y abordar el "problema de raíz” y no remediar únicamente los “síntomas”. Así, cada intervención debe estar en proporción a los criterios de seguridad, manteniendo la intervención al mínimo posible para garantizar la seguridad y durabilidad de la estructura con el mínimo daño posible a los valores intrínsecos. La selección de una técnica de refuerzo debe estar basada en los principios de conservación y los criterios modernos para el análisis y restauración de estructuras históricas, los cuales se encuentran estipulados de forma general en la Carta de Venecia (ICOMOS, 1964) y de forma particular en los criterios ISCARSAH de ICOMOS (2003). Estos principios incluyen los requisitos de diseño que en forma resumida son los siguientes:

- Respeto a la autenticidad estructural. Es decir, la concepción estructural de los edificios históricos es parte de su valor cultural, por lo que es necesario preservar tanto el sistema original como sus materiales.

- Mínima alteración o impacto. Las intervenciones que causen la mínima alteración o impacto al sistema estructural original deberían de preferirse, siempre y cuando provean un nivel de seguridad adecuado.

- Seguridad estructural. En el caso de monumentos valiosos, las intervenciones deben considerar las pérdidas artísticas o culturales que el edificio puede experimentar en caso de daño estructural.

- Compatibilidad. Los materiales y los dispositivos técnicos utilizados para reparación o refuerzo deben ser compatibles con los originales, es decir, que ningún efecto indeseable debería resultar de su utilización. Los materiales antiguos no deberían experimentar algún tipo de deterioro químico cuando esté en contacto con los materiales nuevos (compatibilidad química), y los materiales nuevos no deberían experimentar fenómenos químicos o físicos que puedan causar algún daño a los materiales existentes.

- No sea invasor. Se deberían preferir reparaciones que sean lo menos invasores posibles, dado que esto contribuye a preservar la integridad de las estructuras (primer requisito). Entre varias alternativas, se debería dar preferencia a la que presente una invasión mínima.

- Reversibilidad y remoción. Siempre que sea posible, las medidas adoptadas deben ser reversibles. Es decir, que al desmantelarlas, el material original o la estructura regresan al estado en que estaba antes, sin sufrir daño o deterioro permanente. Un requisito menos rígido es el de la remoción con deterioro limitado o duradero en la construcción original. La reversibilidad o remoción abre la posibilidad de reponer o cambiar, en el futuro, el refuerzo por otro más adecuado o efectivo.

- Monitorización. Debe ser posible controlar la intervención durante su ejecución. No se deberían permitir acciones que sean imposibles de controlar. Un programa de supervisión y control debería acompañar a cualquier propuesta de intervención, con el fin de evitar dañar a la estructura durante los trabajos de intervención. 


\section{ESTRATEGIA INTEGRAL DE ANÁLISIS COMPLEMENTARIOS}

Con base en lo anterior, se observa que en un proyecto de intervención es necesario realizar un análisis integral del edificio histórico. Por análisis se debe entender "el estudio de los límites, características y posibles soluciones de un problema” (RAE, 2001). Por lo tanto, la estrategia de análisis aquí propuesta no está referida únicamente al análisis numérico de la estructura, como se sobrentiende comúnmente en ingeniería, sino que está referida al análisis que se lleva a cabo para la comprensión total del comportamiento estructural de la edificación, con el fin de evitar la pérdida de los valores intrínsecos de la estructura. En este contexto, el análisis y modelado numérico se subordina a un proceso de "análisis" más general, y por ende más completo y complejo de la estructura en el cual intervienen otros factores a parte de los puramente numéricos. Así, el objetivo central de la estrategia de análisis se convierte en el entendimiento global de la estructura, el cual hace que la estrategia de análisis sea integral. Con base en esto, los análisis que se deberán desarrollar y que se complementarán entre sí son:

- Análisis de las Condiciones Pasadas. Contribuye a conocer la historia de la edificación a lo largo del tiempo. Permite identificar los valores intrínsecos del inmueble, así como ayuda a entender la condición actual del mismo. Haciendo una analogía médica, este análisis sería el equivalente de tener la historia médica del paciente; sin la cual sería difícil hacer un diagnóstico correcto de su situación actual.

- Análisis de las Condiciones Actuales. Las condiciones presentes permiten saber, precisamente, el grado de afectación del inmueble. Es aquí donde se planea la posible intervención a realizar.

- Análisis de las Condiciones Futuras. Es necesario que se reconozca la importancia de tener un análisis o previsión de las condiciones futuras del inmueble. La importancia radica en que uno de los objetivos de la conservación es la de prever las posibles acciones a futuro, para permitir que el inmueble se mantenga vivo, conserve su identidad original y se evite la pérdida de sus valores intrínsecos.

\section{Análisis de las condiciones pasadas}

En el análisis de las condiciones pasadas de la estructura se recolecta toda la información útil para desarrollar los dos análisis siguientes. La información debe incluir, entre otros: información histórica, materiales, descripción geométrica y estructural, estudios preliminares. La información histórica es de suma importancia pues ella nos llevará a entender el comportamiento estructural del edificio histórico a lo largo del tiempo, principalmente ante eventos extraordinarios como son: sismos, hundimientos diferenciales, huracanes, etc. De especial interés es la descripción histórica de los daños presentados, así como de las distintas reparaciones a las que ha sido sometido, como por ejemplo: cambios de materiales, modificaciones estructurales y arquitectónicas, cambios de uso y por lo tanto de cargas, entre otros.

Entre otro tipo de información que este análisis debe proporcionar está:

- Información de las edificaciones que hayan ocupado el predio. Contribuye a conocer el grado de consolidamiento del suelo, posibles restos de edificaciones pasadas, etc.

- Edificación original. Es decir tipos de materiales usados, cimentación, estilo arquitectónico, etc.

- Análisis cronológico. Se deben mostrar los daños, modificaciones y supresiones que el edificio haya tenido a lo largo de su historia

- Usos de la edificación. Debido a que se pudo haber modificado el uso de la estructura y por lo tanto de las cargas. 
- Recopilación de información. La información de todo tipo ayuda a tener un mejor entendimiento del comportamiento de la estructura. Esta recopilación debe incluir material fotográfico, planos, diversos tipos de levantamientos, pinturas, grabados, etc.

\section{Análisis de las condiciones actuales}

El análisis de las condiciones actuales de la estructura permite conocer el grado de afectación de la misma. Estas condiciones se ven complementadas tanto por el estudio histórico, como por el arquitectónico. Es de suma importancia contar con una buena descripción geométrica y estructural, ya que en general, la geometría estructural de las construcciones históricas es bastante compleja, por lo que muchas veces no hay una clara diferencia entre los elementos estructurales y los arquitectónicos.

\section{Análisis de las condiciones futuras}

Este análisis se refiere a las cargas que deberá resistir la estructura en el futuro; por ejemplo sismo, hundimientos diferenciales, degradación de los materiales, flujo plástico, entre otras. En este caso, la realización de análisis paramétricos siempre es valiosa para poder reducir las incertidumbres derivadas de las variabilidades de un parámetro (por ejemplo registros sísmicos) o a la falta de información o idealización realizada (degradación de los materiales, flujo plástico).

\section{ESTRATEGIA INTEGRAL DEL MODELADO Y EL ANÁLISIS NUMÉRICO}

El análisis "cuantitativo" del estado de la estructura (tanto pasado, presente, como futuro) forma parte del proceso de diagnóstico y evaluación estructural. Este análisis cuantitativo se basa principalmente en estudios analíticos. Con base en esto, se proponen una serie de pasos que se deben ir completando con el fin de superar las dificultades inherentes al análisis numérico de las estructuras históricas; los cuales son:

- Adquisición de datos

- Selección de las herramientas de análisis

- Proceso de calibración y validación

- Selección de los tipos de análisis numéricos

- Análisis paramétricos

\section{Paso 1 - Adquisición de datos}

En esta etapa se recolecta toda la información disponible. La cual incluye, entre otros: información histórica, materiales, descripción geométrica y estructural, estudios preliminares, etc. En general, la geometría estructural de las construcciones históricas es bastante compleja, por lo que muchas veces se confunden los elementos arquitectónicos con los estructurales. Debido a esto es importante contar con una buena descripción geométrica y estructural. Así mismo, a diferencia de las estructuras modernas, no es posible definir en modo general una idealización de la geometría, sea ésta con elementos barra, placa o sólidos. Esta idealización dependerá fundamentalmente del tipo de elemento estructural y su geometría, pero también dependerá de sus condiciones de frontera, apoyos, solicitaciones y de la idealización de otros elementos.

La caracterización mecánica del material se da en esta etapa. Como la mampostería es un material de construcción compuesto por bloques unidos por juntas, en un modelo analítico de una estructura real, 
resulta impráctico, sino imposible, la discretización de cada bloque y junta de mortero. Entonces, es necesario considerarla como un material homogéneo con el cual se describe el comportamiento heterogéneo de la mampostería al tomar en cuenta las propiedades medias del material (como son las constantes elásticas y los parámetros de resistencia). A este proceso se le conoce como homogenización que se puede obtener mediante pruebas numéricas con un modelo computacional (generalmente de elementos finitos) o bien directamente mediante pruebas experimentales (sean en laboratorio o in-situ).

El comportamiento mecánico de la mampostería puede ser tomado en cuenta considerando las propiedades medias del material usando la teoría de homogenización para materiales periódicos. En general, la identificación de los parámetros mecánicos de la mampostería puede ser obtenida mediante un modelo computacional, en el cual se considera la respuesta de una célula básica en la cual se incluyen todos los materiales (Anthoine, 1995; Figura. 1).

a)
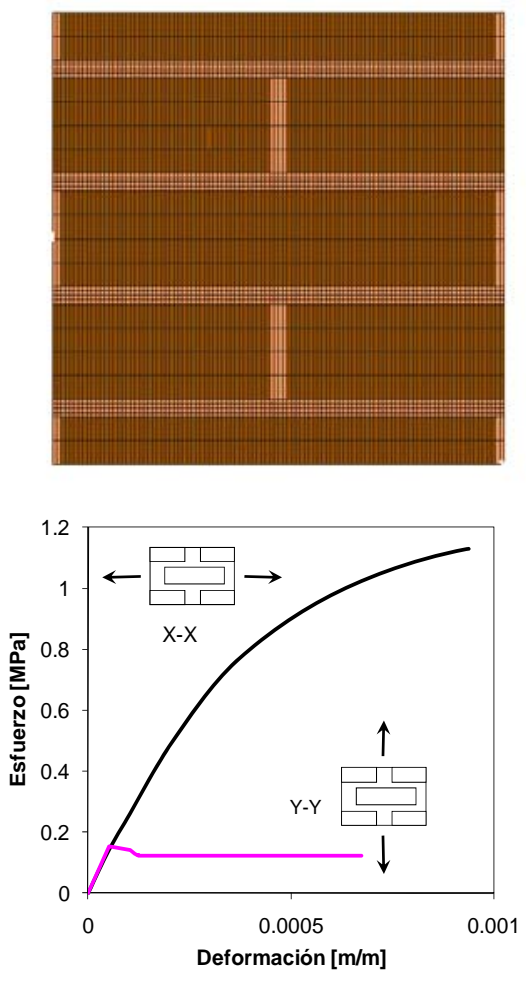

c) b)
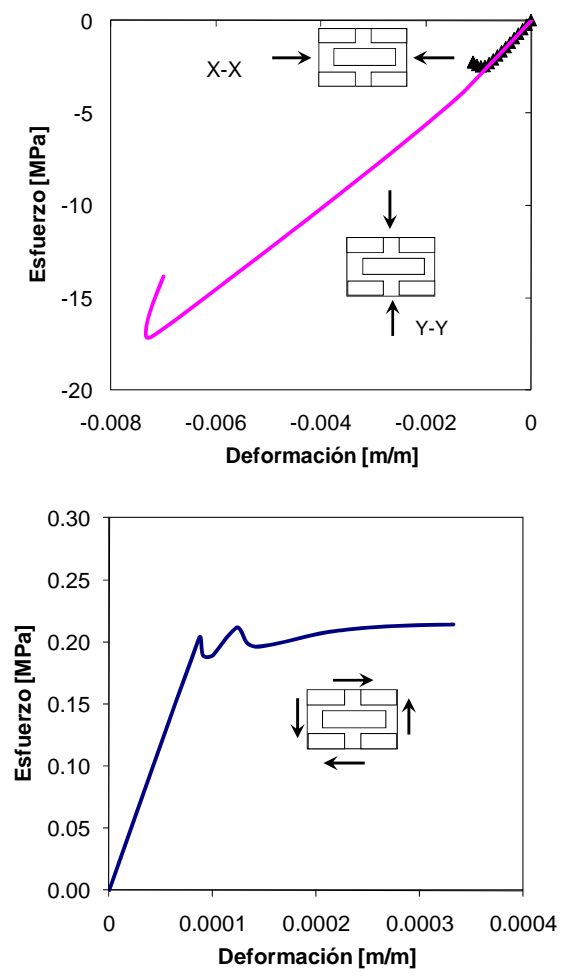

Figura 1. Ejemplo de homogenización de una mampostería típica mediante un modelo de elementos finitos, en el que se modelan tanto las piezas como las juntas: a) modelo; b) respuesta a compresión; c) respuesta a tensión; d) cortante

Generalmente, este tipo de homogenización se hace con modelos en dos dimensiones. Cuando se trabaja con elementos finitos en dos dimensiones es común utilizar elementos sólidos planos. La formulación de estos elementos puede ser mediante deformaciones o esfuerzos planos. La hipótesis de deformaciones planas es exacta cuando el espesor del elemento a analizar tiende a infinito. Por otro lado, la hipótesis de esfuerzos planos es exacta cuando el espesor tiende a cero. Sin embargo, cuando se estudia un elemento con un espesor finito ambas suposiciones no son del todo correctas; pues los esfuerzos planos subestiman la respuesta mientras que las deformaciones planas la sobrestiman (Pegon y Anthoine, 1997). 
Debido a esto, es común utilizar la formulación de deformaciones generalizadas planas (Anthoine, 1997). La diferencia con la formulación de las deformaciones planas es que las primeras permiten deformación fuera del plano (axial, flexión y torsión). Sin embargo, estas deformaciones permanecen constantes a lo largo del eje fuera del plano y no modifican las ecuaciones diferenciales de la formulación clásica de deformaciones planas. Generalmente, estas deformaciones se obtienen considerando la fuerza resultante aplicada perpendicular al plano igual a cero. Esto significa que las caras del elemento a analizar no están cargadas (Pegon y Anthoine, 1997).

Cuando sea posible, se recomienda la realización de estudios preliminares, fundamentalmente pruebas no destructivas realizadas in-situ. Por ejemplo, la determinación de los modos de vibrar de la estructura mediante vibración ambiental ayuda a la posterior calibración de los modelos analíticos (Peña et al., 2010). Las pruebas de georadar permiten observar cambios de material, oquedades, etc. (Binda et al., 2007).

\section{Paso 2 - Selección de las herramientas de análisis}

Existen diversos métodos y modelos para estudiar edificios históricos. Por una parte, están los métodos muy refinados como los métodos de elemento finitos (Lourenço, 2002), discretos o discontinuos (Lemos, 2007). Por otro lado, están los modelos simplificados como son los modelos de macroelementos (Brencich y Lagomarsino, 1998), modelos de elemento rígidos (Casolo y Peña, 2007) o análisis límite (Orduña y Lourenço, 2005). La utilización de cada método depende del problema y los recursos para resolverlo. En general los métodos refinados permiten analizar estructuras complejas, describiendo correctamente los materiales. Pero los recursos computacionales necesarios son elevados, por lo que muchas veces no es posible hacer el modelado de estructuras completas o realizar análisis paramétricos. Por otra parte los métodos simplificados son útiles para analizar grandes estructuras o realizar análisis paramétricos, pero ellos tienen la limitante de sus hipótesis simplificadoras que algunas veces pueden no cumplirse.

A continuación se describirán brevemente tres métodos y dos tipos de análisis utilizados en el análisis de construcciones históricas. Éstos se seleccionaron con base en que logran cubrir una amplia gama de métodos y análisis utilizados en la práctica e investigación:

- Elementos Finitos. Este método logra definir cualquier tipo de geometría, tanto en dos o tres dimensiones. Permite una buena definición de las características de los materiales y solicitaciones. Es adecuado para cualquier tipo de estructura que se pueda considerar continua (Fig. 2), así como también su formulación es ampliamente conocida. Sin embargo, este método demanda una gran cantidad de recursos computacionales que muchas veces no están disponibles. Además de que algunas veces los parámetros para definir las propiedades inelásticas de los materiales son difíciles de evaluar, como la energía de fractura.

- Elementos Discretos. Este método es idóneo en el estudio de medios discontinuos, ya que permite el movimiento relativo entre elementos, así como el cambio de geometría la cual se calcula de forma automática. Un ejemplo de este tipo de estructuras son las construcciones formadas con bloques de piedra como columnas, esculturas, arcos, templos griegos o romanos (Fig. 3). Sin embargo, las características mecánicas de las juntas entre elementos son difíciles de evaluar; así como la solución numérica es lenta, debido a que considera cambios en la geometría.

- Elementos Rígidos. Es un método sencillo, eficaz y particularmente rápido para el estudio dinámico no lineal, dado que son necesarios pocos elementos, aún para geometrías complejas (Fig. 4). Sin embargo, presenta la limitante de que permite solamente el análisis en dos dimensiones, así como su formulación no es muy conocida. 
- Análisis Límite. Es un análisis adecuado para fines de diseño, por su rapidez y sencillez del análisis. Únicamente se puede conocer el mecanismo de colapso y la carga última (Fig. 5).

- Análisis Elástico - Lineal. Es un análisis sencillo, rápido y ampliamente utilizado por los ingenieros de la práctica. Permite conocer las zonas débiles de la estructura, así como los elementos que puedan presentar un comportamiento indeseable o poco satisfactorio. Sin embargo, sólo es recomendable como análisis preliminar, ya que sobrestima fuerzas y esfuerzos, y subestima desplazamientos y deformaciones. Al no tomar en cuenta el daño de los elementos, no considera la redistribución de fuerzas y esfuerzos. En algunos casos esta redistribución puede ser causa de daños en otras partes de la estructura.

Como se ha visto, existen varios métodos y herramientas computacionales para la evaluación del comportamiento mecánico de construcciones históricas. La selección de la herramienta de análisis no es una cosa trivial, pues de ella dependerá en gran medida la validez de los resultados obtenidos. Cabe hacer notar que el hecho de que una herramienta de análisis sea más compleja que otra, no necesariamente proporcionará mejores resultados que una herramienta simplificada. Los aspectos que se deberían tomar en cuenta para seleccionar una herramienta de cálculo son (Lourenço, 2002):

- Información disponible. Es necesario que los datos que se dispongan de la estructura, así como de las solicitaciones actuantes, sean suficientes para poder definir el modelo matemático. Estos datos son recopilados en el paso anterior: adquisición de datos. La falta de información se podría suplir con datos obtenidos de la literatura o tomando valores nominales. Sin embargo, esto involucraría incertidumbres adicionales al problema.

- Resultados esperados. Los resultados que se puedan obtener del método de análisis deben proporcionar la información requerida para el proyecto. Algunas veces, las limitaciones intrínsecas de la herramienta de análisis no permiten obtener la información necesaria. Por ejemplo, un análisis límite no permite conocer las deformaciones de la estructura o un análisis en dos dimensiones no podrá considerar efectos tridimensionales como son las torsiones en algunos elementos.

- Disponibilidad y Compatibilidad. Obviamente la herramienta de análisis debe ser de fácil acceso y manejo (disponibilidad) para el profesionista encargado del análisis. Así mismo, es de vital importancia que dicho profesionista tenga el conocimiento suficiente de la teoría en que está basado el método de análisis (compatibilidad) para poder hacer una correcta modelación e interpretación de los resultados
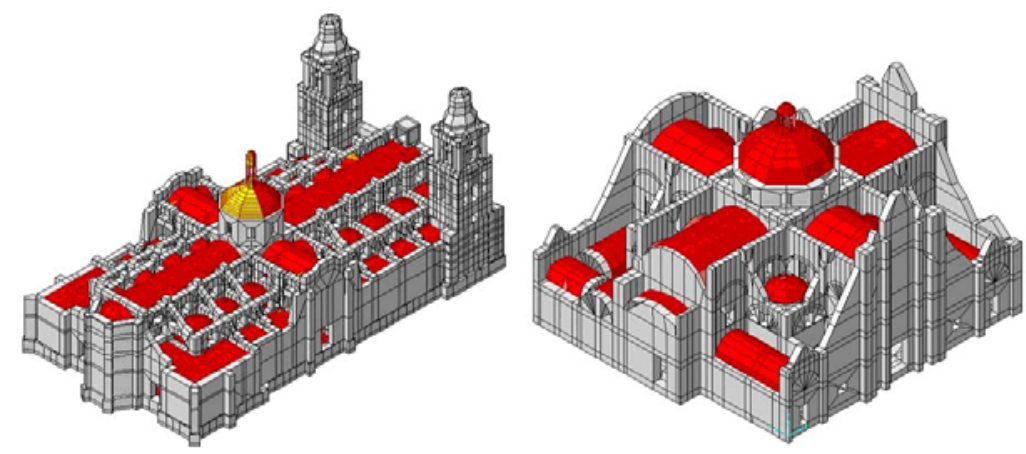

Figura 2. Modelos de elementos finitos de la Catedral y el Sagrario de la ciudad de México (Meli y Sánchez, 1995) 
a)

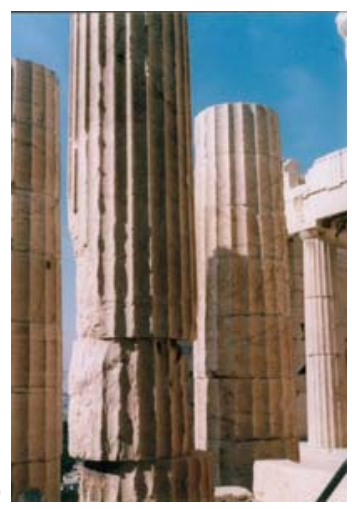

b)

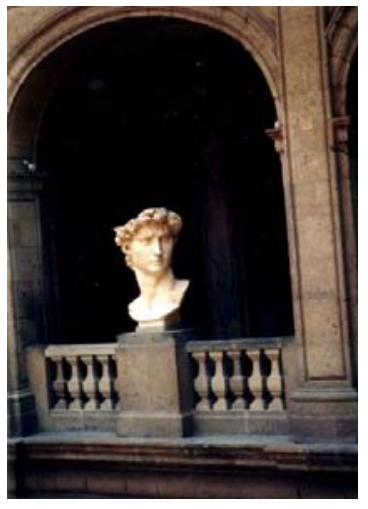

Figura 3. Ejemplo de estructuras que deben modelarse con elementos discretos: a) estructuras formadas con bloques de piedra; b) objetos de arte

a)

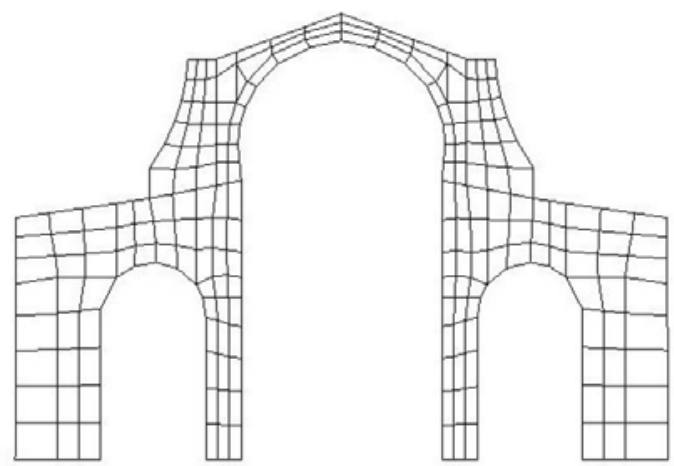

b)

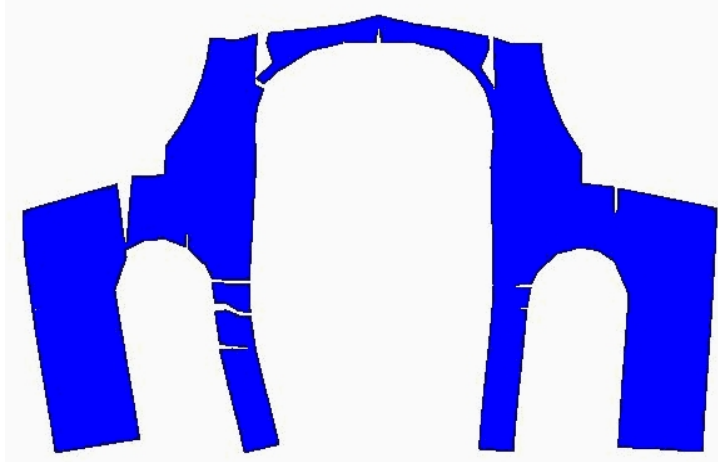

Figura 4. Ejemplo de un modelo de una arcada de la Catedral de Noto, Sicilia, Italia: a) modelo de elementos rígidos; b) mecanismo de colapso (Peña y Casolo, 2003)

a)

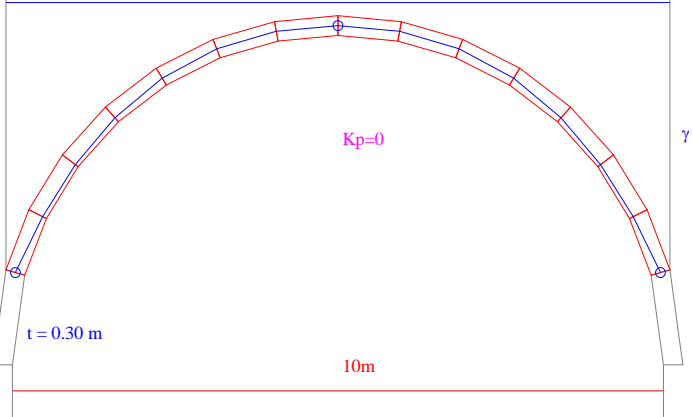

b)

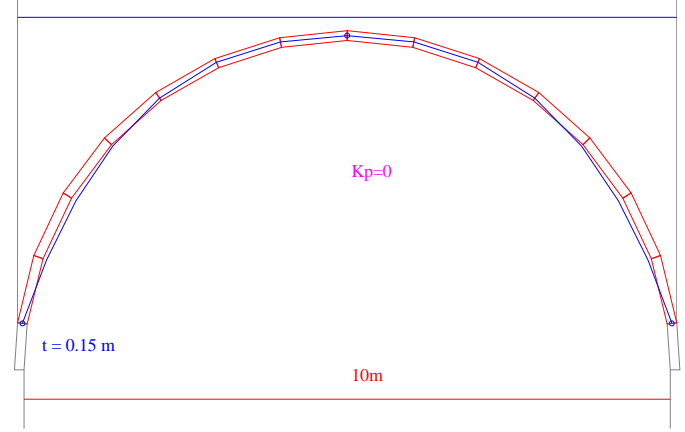

Figura 5. Ejemplo de dos arcos estudiados con análisis límite: a) arco estable; b) arco inestable

- Costos. Se debe de tomar en cuenta que los recursos disponibles estén de acuerdo con los requeridos por la herramienta de análisis. Los recursos son tanto financieros, como de capacidad de cálculo (cómputo), tiempo disponible para el análisis e interpretación de resultados, entre otros.

- Idealización y Validación. Todo modelo analítico se basa en la idealización de la realidad, por lo tanto, es necesario que sea lo más apropiada posible. En general se hace una idealización de los elementos estructurales, el comportamiento y características del material, las solicitaciones 
actuantes. Así mismo, el modelo matemático resultante de la idealización de la realidad, debe ser validado con el fin de asegurarse de que la idealización sea correcta. Esta validación se da en el paso tres, como se explica en la siguiente sección.

Por otro lado, muchas veces, una sola herramienta de análisis no es suficiente para cubrir satisfactoriamente todos los aspectos requeridos. Por ejemplo, los análisis tridimensionales no-lineales de estructuras completas, generalmente, no son posibles de realizarse por la gran cantidad de recursos computacionales que requieren. Otro ejemplo, sería que algunas veces las hipótesis en las que se basan los modelos simplificados no se cumplen cabalmente, por lo que su uso no es recomendable.

Debido a esto, el enfoque integrado de análisis complementarios lo que busca es el uso de dos o más herramientas de análisis. De este modo, se utilizan los resultados obtenidos a partir de una herramienta de análisis como resultados preliminares o para una mejor concepción de otro modelo utilizando una herramienta de análisis diferente. De aquí el nombre de análisis complementarios. Es decir, los análisis realizados con diferentes técnicas o herramientas de análisis se "complementan", para obtener una respuesta más completa y satisfactoria de la estructura a analizar.

Generalmente, una propuesta que ha dado buenos resultados es la de usar modelos simplificados en combinación con modelos refinados (Peña et al., 2007). Los resultados obtenidos de un modelo sirven de base para una mejor concepción del otro modelo, el cual puede utilizar una herramienta de análisis diferente. Así mismo, combinando los resultados de los diferentes modelos, es posible obtener una mejor y más completa estimación del comportamiento estructural de un edificio histórico.

Cuando se analizan iglesias por sismo es común dividirlas en macroelementos. Los cuales se pueden definir como la parte de una iglesia cuya respuesta sísmica puede ser evaluada en forma independiente del resto de la estructura. De este modo se evita tener un modelo numérico de toda la iglesia, reduciendo así el costo computacional al tener modelos más pequeños de partes de la estructura original (Fig. 6). La idea de los macroelementos surge cuando se presentan mecanismos de daño. Éstos se forman cuando el daño en la estructura crea secciones con comportamientos similares al de cuerpos rígidos. De esta forma, el daño actúa en forma de articulación o desliga el elemento del resto de la estructura (Doglioni et al., 1994).

\section{Paso 3 - Proceso de calibración y validación}

Cabe recordar que, todo análisis estructural se realiza haciendo una idealización de la realidad, sea ésta la geometría, el comportamiento del material y la estructura, las solicitaciones actuantes, etc. Obviamente, es necesario que las idealizaciones elegidas sean apropiadas al problema en consideración, de modo que, el modelo matemático resultante sea capaz de representar la realidad que está simulando.

En este nivel se hace un proceso de calibración y validación, el cual se puede realizar con pruebas experimentales (in situ o laboratorio), con otros modelos matemáticos, con resultados obtenidos de la literatura, etc. Esto dependerá de la cantidad de información recopilada en el primer paso. Así mismo, es necesario que los modelos se validen ante las diferentes solicitaciones a las cuales será sometido el modelo. Naturalmente, éstas dependerán de los tipos de análisis que se llevarán a cabo (paso cuatro). Cada modelo tendrá que ser evaluado en forma independiente.

En forma general, los análisis de peso propio y cálculo de frecuencias y modos de vibrar se pueden utilizar como una forma sencilla de calibración y validación preliminar de los modelos numéricos. El peso propio de una estructura se puede calcular de forma sencilla el cual se compara con el obtenido con el modelo numérico. Por otro lado, el cálculo de las frecuencias y modos de vibrar permite conocer que la 
relación entre masas y rigideces es la adecuada. Con este análisis también, es posible detectar algunos errores en el mallado.

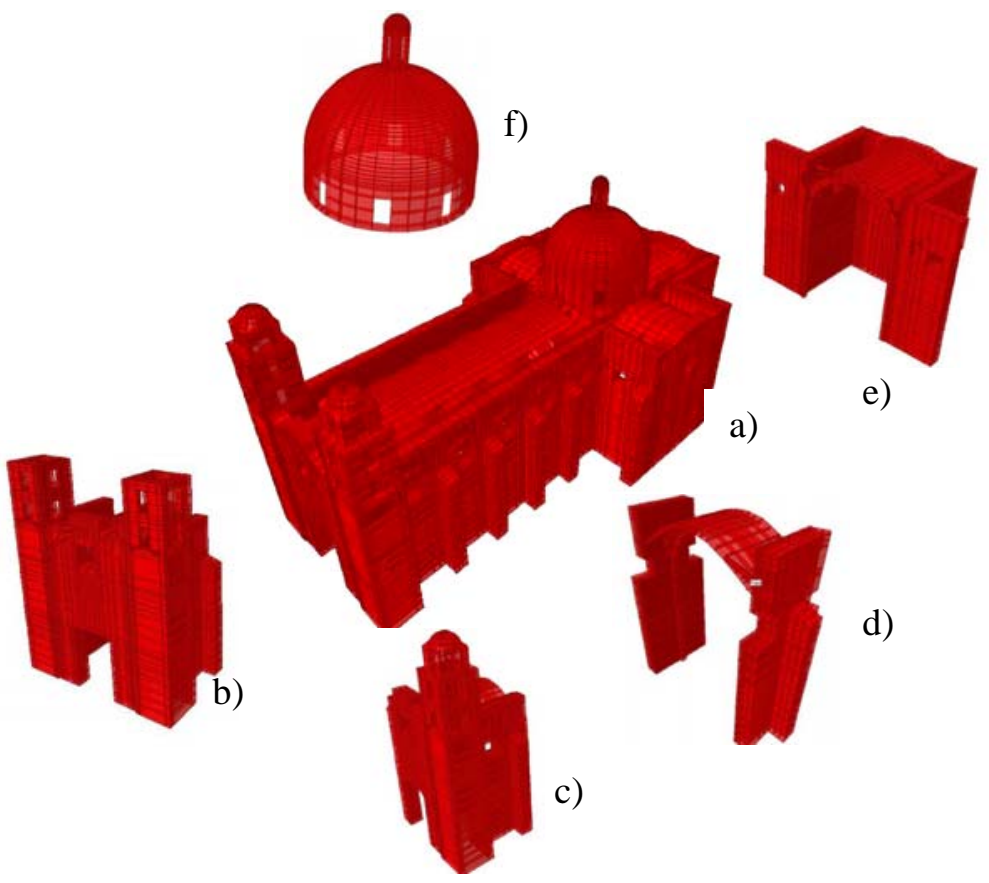

Figura 6. Macroelementos típicos de una iglesia en forma de cruz latina: a) modelo de la iglesia completa;

b) macroelemento fachada; c) macroelemento torre de campanario; d) macroelemento nave; e) macroelemento ábside; f) macroelemento cúpula

Una forma de obtener las propiedades elásticas de los materiales puede ser mediante la calibración de las frecuencias y modos de vibrar del modelo analítico con respecto a pruebas de vibración ambiental. Esto se puede lograr utilizando la metodología propuesta por Douglas y Reid (1982), mediante la cual, las frecuencias de una estructura se pueden calcular por medio de:

$f_{i}^{D}\left(X_{1}, X_{2}, . ., X_{N}\right)=C_{i}+\sum_{i=1}^{N}\left[A_{i k} X_{k}+B_{i k} X_{k}^{N}\right]$

donde $f^{D}$ es la frecuencia estimada, $X_{k}$ son las variables a calibrar, $A, B, C$, son constantes, y $N$ es el número de frecuencias usadas en la calibración. Así, las variables son obtenidas mediante la optimización de la función $J$ :

$$
\begin{aligned}
& J=\sum_{i=1}^{m} w_{i} \varepsilon_{i}^{2} \\
& \varepsilon_{i}=f_{i}^{E M A}-f_{i}^{D}\left(X_{1}, X_{2}, \ldots, X_{N}\right)
\end{aligned}
$$

donde $f^{E M A}$ es el valor experimental de las frecuencias y $w$ es un factor de peso. 
a)

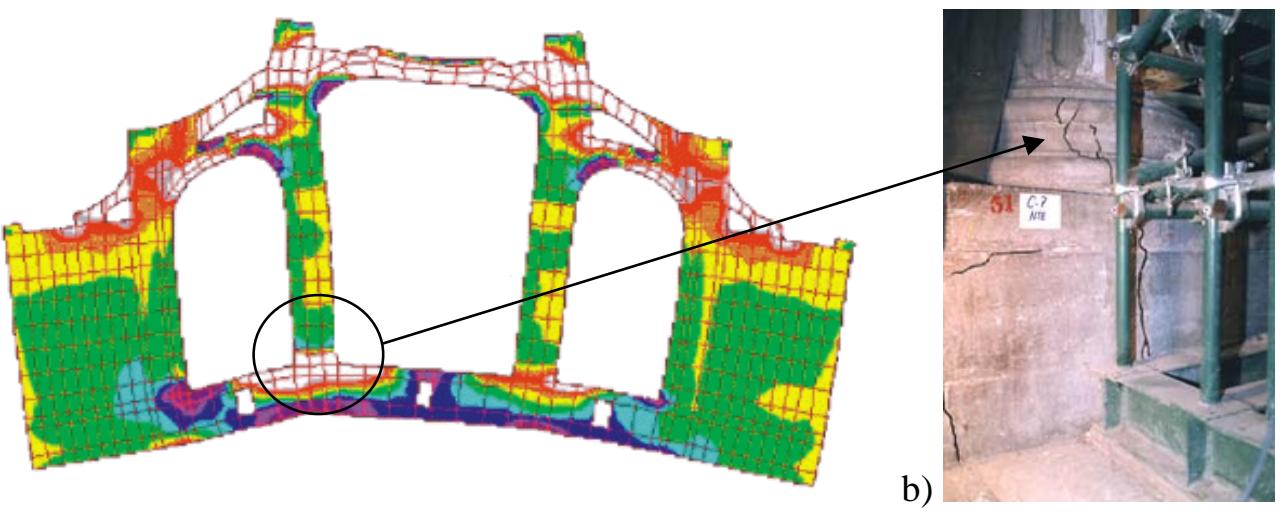

Figura 7. Ejemplo de la validación de un modelo confrontándolo con los daños que presenta la estructura:

a) modelo de elementos finitos; b) daño real en la base de una columna (Meli y Sánchez, 1995)

Por otro lado, considerando los daños presentes en la estructura por analizar, se puede hacer una calibración y validación del modelo de una forma cualitativa. Es decir, se puede validar el modelo al lograr reproducir los daños que presenta la estructura. Por ejemplo, la Figura 7 presenta un modelo de elementos finitos de una arcada de la Catedral de la ciudad de México, el cual fue sometido a una condición de hundimientos diferenciales. En este caso, se realizó la validación del modelo confrontándolo con los daños que presentaba el templo. Nótese que el agrietamiento real en la base de una columna debido a los hundimientos diferenciales, el cual coincide con las zonas de tensión (color blanco) que aparecen en el modelo, precisamente en la base de la columna (Meli y Sánchez, 1995).

\section{Paso 4 - Tipos de análisis}

La selección del tipo de análisis dependerá de la herramienta de análisis escogida, de los costos en general (recursos computacionales disponibles, de los tiempos de pre y posproceso, etc.), así como también del tipo de estructura histórica que estemos analizando. También, es importante la forma en cómo se idealizarán las solicitaciones: sean del tipo estático, dinámico, modal espectral; sean fuerzas, desplazamientos, aceleraciones, etc. Dependiendo del fenómeno a estudiar será la forma de idealizar las solicitaciones.

En forma general, se pueden dividir los análisis en elásticos y en no lineales. Como se comentó anteriormente, ningún estudio sobre el comportamiento estructural de un edificio histórico se debería basar única y exclusivamente en análisis elástico lineales, pues se corre el riesgo de no tener una correcta valoración del comportamiento estructural. Por lo que el uso de métodos inelásticos se torna indispensable. Sin embargo, algunas veces los parámetros necesarios para definir correctamente el modelo matemático que describe el comportamiento no-lineal del material son difíciles de evaluar. Por ejemplo, varios modelos requieren la energía de fractura del material, dato que no es sencillo de encontrar experimentalmente, ni tampoco en la literatura existiendo pocos datos referentes al tema.

Así mismo, dependiendo del fenómeno a estudiar será la forma de idealizar las solicitaciones. Por ejemplo, los hundimientos diferenciales se pueden estudiar como desplazamientos predeterminados en la base (Fig. 7). Los sismos se pueden estudiar con análisis estáticos o dinámicos, sin embargo, como se comentó anteriormente, los análisis inelásticos son indispensables, independientemente de cómo se idealicen las solicitaciones. 
Cuando se analiza una estructura histórica se recomienda que se utilicen análisis dinámicos, en lugar de los estáticos; sobre todo cuando se tiene una geometría compleja. Esto se debe a que muchas veces los modos superiores de vibrar influyen significativamente en el comportamiento global de la estructura, por lo que, la estructura histórica rara vez se puede simplificar como un sistema de un grado de libertad.

Para ejemplificar esto, considere el macroelemento fachada de una iglesia típica de Oaxaca analizada con un modelo de elementos rígidos (Peña y Meza, 2008). La Figura 8a muestra el mecanismo de colapso obtenido con un análisis estático no-lineal considerando fuerzas proporcionales a las masas de los elementos (análisis del empujón o pushover). El daño que se presenta es una grieta vertical que cruza la puerta y la ventana del coro, lo que provoca que la fachada se divida en dos. De este modo, cada torre permanece adosada a una parte de la fachada las cuales presentan una flexión generalizada. Por otro lado, el daño obtenido con un análisis dinámico no-lineal (Fig. 8b) se concentra en los campanarios, así como en el cuerpo de la torre. Estos últimos daños coinciden con los daños registrados en estas estructuras después del sismo del 15 de junio de 1999 (Fig. 8c).

Así mismo, los análisis estáticos no-lineales predicen resistencias mayores que las obtenidas con los análisis dinámicos. Esto se debe a que los análisis estáticos no toman en cuenta la influencia de los modos superiores de vibrar, en este caso, los campanarios tienen un comportamiento tipo apéndice que incrementa las aceleraciones. Por lo tanto, no se recomienda el uso de análisis estático lineales para el estudio de torres de campanario; siendo necesario el uso de análisis dinámicos no-lineales. Finalmente, obsérvese que la validación de estos resultados se hizo comparando los daños observados con los daños obtenidos (aplicación del paso 3); desechando los resultados del análisis estático, al no representar correctamente la realidad.

a)

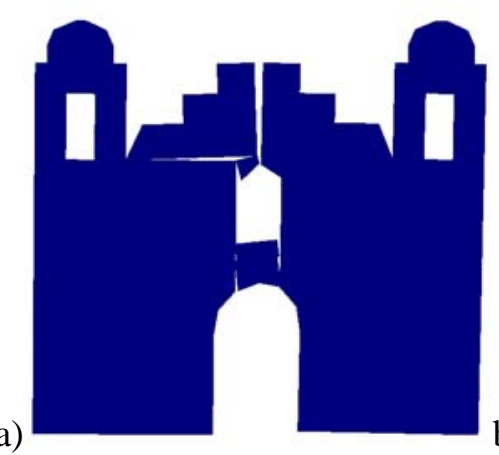

b)

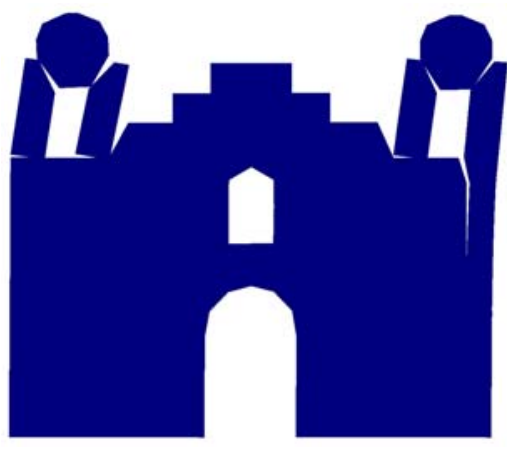

c)

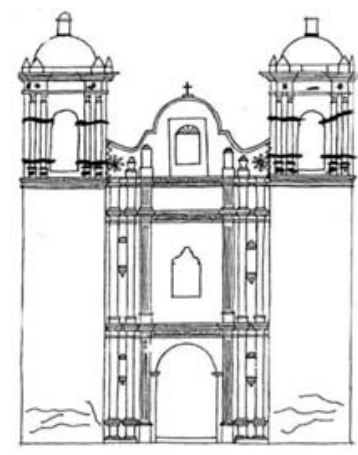

Figura 8. Mecanismo de colapso de la fachada de una iglesia típica de Oaxaca: a) análisis estático nolineal o análisis del empujón (pushover); b) análisis dinámico no-lineal; c) daños típicos observados después del sismo del 15 de junio de 1999 (Peña y Meza, 2008)

\section{Paso 5 - Análisis Paramétricos}

Algunas veces, las idealizaciones realizadas a los materiales o a las solicitaciones, pudieran ser no del todo satisfactorias. Esto se puede deber, principalmente, a la falta de información (paso 1) o a la variabilidad propia del parámetro (por ejemplo registros sísmicos). En estos casos, para disminuir las incertidumbres derivadas de dichas idealizaciones se recomienda la realización de análisis paramétricos. El objetivo de estos análisis es el de evaluar la influencia de alguna variable o parámetro en el comportamiento estructural. 
Para tal efecto, es necesario definir valores base, valores límite superior e inferior. El valor base es aquel con que se inician los análisis y se consideran como representativos de la estructura. Los valores límite determinan el rango de variación que puede tener la variable o parámetro a considerar. Cabe hacer notar que, un valor base puede ser también un valor límite, sea inferior o superior.

Cuando se realiza un análisis dinámico, las cargas se definen como aceleraciones que varían con el tiempo. Estos registros de aceleraciones pueden ser de sismos reales registrados, funciones matemáticas (por ejemplo funciones harmónicas como el seno o coseno), sismos sintéticos generados a partir de un espectro de diseño. En un análisis dinámico, la estructura podrá tener diferentes comportamientos dependiendo del contenido de frecuencias y duración de la señal, del valor máximo de aceleración, velocidad y desplazamiento del suelo, etc. Debido a estas incertidumbres, se hace necesario realizar análisis con distintos tipos de registros, es decir un análisis paramétrico.

Un ejemplo de esto, es el estudio de la vulnerabilidad sísmica de una arcada de la Catedral de Noto, en Sicilia, Italia, (Fig. 4; Peña y Casolo, 2003), la cual fue sometida a tres registros diferentes, dos reales registrados en 1976 durante el sismo de Umbria - Marche, y uno sintético compatible con el espectro de diseño del Eurocódigo 8 (2004). La Figura 9 muestra los espectros de respuesta de estos registros.

La Tabla 1 muestra el cuadro de daño y la deformada máxima, para cada uno de los registros. Se observa que la estructura tiende a dañarse en modo similar para los tres registros, manifestando un daño a tensión en toda la estructura que se incrementa conforme aumenta la intensidad de la solicitación. El daño inicia a manifestarse en el intradós de las bóvedas de las naves laterales y de ahí se difunde hacia el extradós y la parte superior de las pilastras. El daño del arco de la nave central está asociado a la combinación de la solicitación lateral y vertical, mientras que el daño a cortante se concentra exclusivamente en la zona de los arbotantes que contrarrestan el empuje lateral de la bóveda. Cabe hacer notar que no se observaron zonas de daño debido a compresión (Peña y Casolo, 2003).
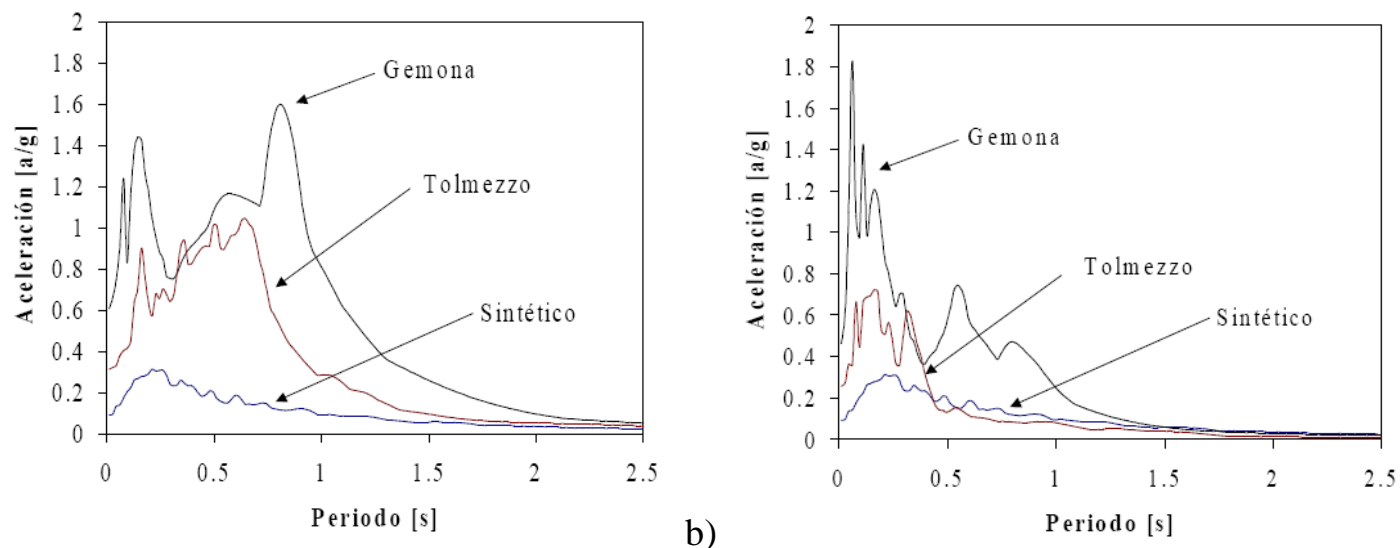

a)

Figura 9. Espectros de respuesta de los tres registros utilizados: a) dirección horizontal (E-W); b) dirección vertical (Peña y Casolo, 2003) 
Tabla 1. Cuadro de daño de una arcada de la Catedral de Noto, Sicilia, Italia (Peña y Casolo, 2003)

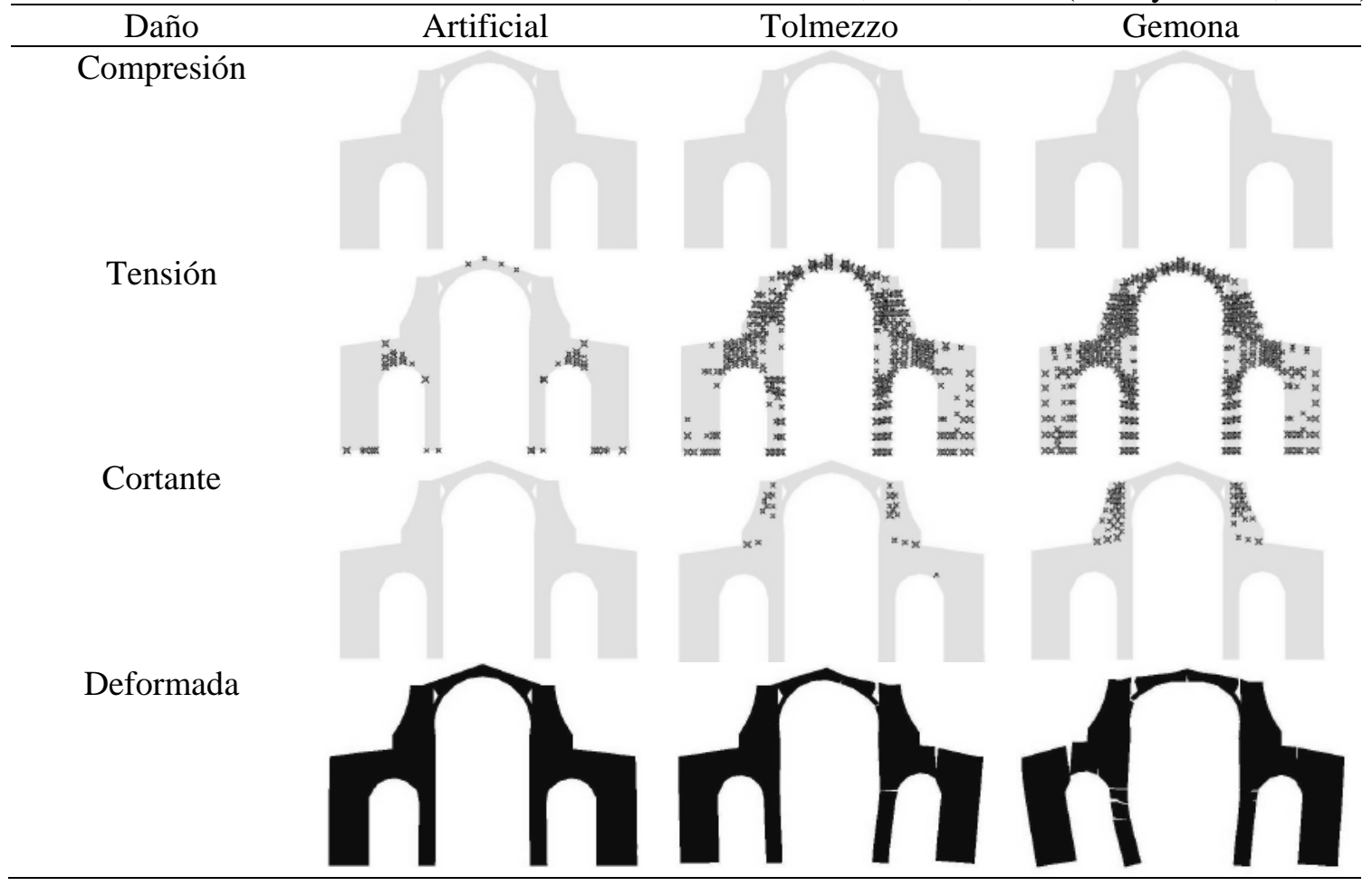

Se realizaron tres modelos diferentes. Dos modelos tridimensionales de elementos finitos, uno de los cuales se realizó usando elementos sólidos (Fig. 10b) y en el otro elementos barra (Fig. 10c). El tercer modelo es en dos dimensiones y se analizó con el método de los elementos rígidos (Fig. 10d). El modelo de elementos sólidos consta de 65,912 elementos, 57,300 nodos y 172,000 grados de libertad. El modelo de elementos barra consta de 20 elementos, 41 nodos y 120 grados de libertad; mientras que el de elementos rígidos consta de 39 elementos, 52 nodos y 117 grados de libertad.

Los tres modelos se calibraron utilizando las pruebas de vibración ambiental realizadas en el minarete (Ramos et al., 2006). El objetivo de estas pruebas fue la determinación de las propiedades dinámicas de la torre (frecuencias y modos de vibrar, así como los amortiguamientos modales). Se obtuvieron 14 modos de vibrar: 10 modos de flexión, dos modos de torsión, un axial y un modo combinado (Fig. 11). Utilizando las ecuaciones 1 a 3 se obtuvieron los módulos de elasticidad de los diferentes materiales que constituyen el minarete.

El modelo tridimensional de elementos sólidos sólo se utilizó para validar las hipótesis simplificadoras de los modelos de elementos barra y rígidos: las escaleras son lo suficientemente rígidas como para considerar que el núcleo central y el cascarón exterior trabajan como una sola sección; y las aberturas no influyen en el comportamiento global de la torre (ver Fig. 10b). Esto se debió principalmente, al costo computacional que involucraba el modelo de elementos sólidos. Cabe hacer notar que el modelo de elementos rígidos requiere de 20 minutos para realizar un análisis dinámico no-lineal, el modelo de elementos barra requiere, para el mismo análisis, de 24 hrs; mientras que el modelo tridimensional hubiera requerido aproximadamente tres meses.

De esta manera, una vez comprobadas que las hipótesis simplificadoras de los modelos de elementos barra y rígidos eran válidas, se realizaron análisis no-lineales, tanto estáticos (pushover) como 
dinámicos paso a paso. Por la velocidad de cálculo, el modelo de elementos rígidos se uso para hacer análisis preliminares, con el fin de observar el comportamiento de la torre. De los análisis estáticos no lineales se observa que la base de la torre es la zona más vulnerable (Fig. 12a). Sin embargo, los análisis dinámicos no lineales determinaron que las zonas críticas son los dos últimos niveles de la torre (Fig. 12b,c). Estos resultados coinciden con los datos históricos disponibles, los cuales muestran que estos dos niveles del minarete han sido los que más daños han sufrido en diferentes eventos sísmicos (Peña et al., 2010). De este modo, los resultados numéricos se validan al compararlos con el comportamiento histórico de la torre ante eventos sísmicos. Así mismo, esta comparación muestra la importancia del análisis de las condiciones pasadas de la estructura, así como del Paso 1 - adquisición de datos.

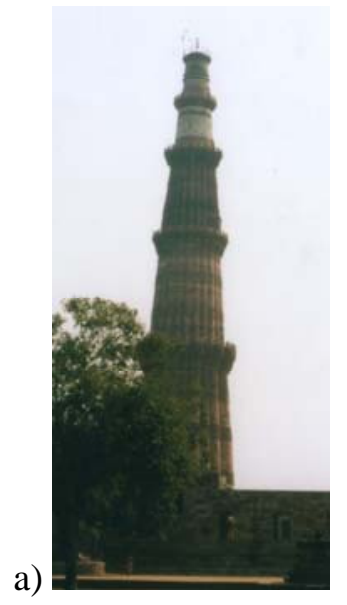

b)

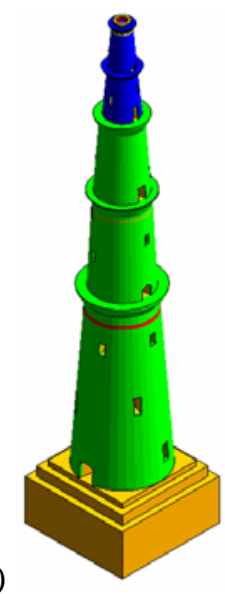

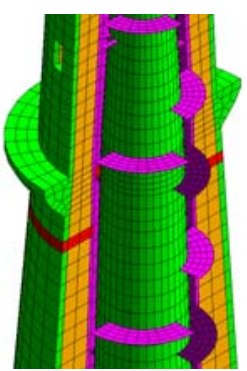

c)

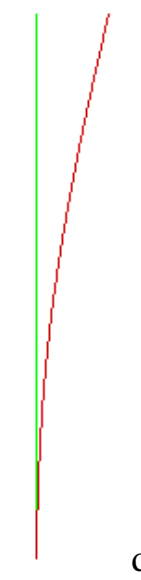

d)

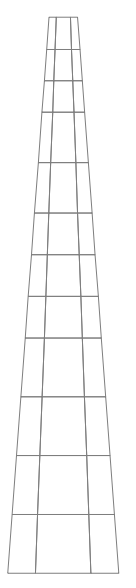

Figura 10. Ejemplo del modelado de una torre de mampostería mediante la estrategia integral de análisis y modelado numérico: a) estructura original; b) modelo de elementos finitos usando elementos sólidos; c) modelo de elementos finitos con elementos barra; d) modelo de elementos rígidos (Peña et al., 2010)

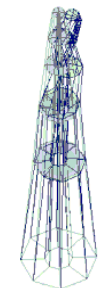

Modo 1

(0.793)

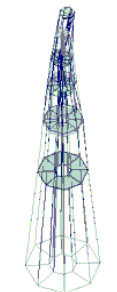

Modo 8

(6.006)

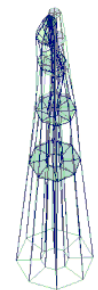

Modo 2

(0.814)

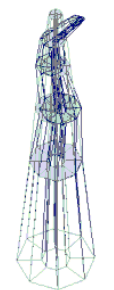

Modo 9

(6.146)

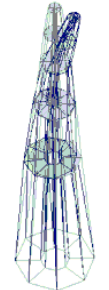

Modo 3

(1.955)

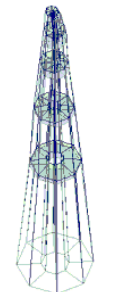

Modo 10

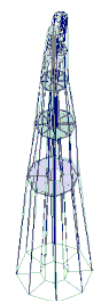

Modo 4

(2.010)

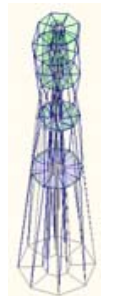

Modo 11

(6.977)

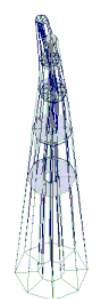

Modo 5

(3.741)

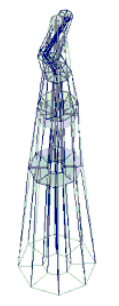

Modo 12

(8.090)

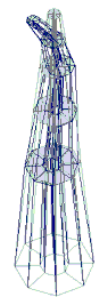

Modo 6

(3.861)

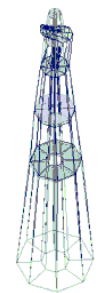

Modo 13

(8.525)

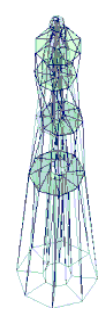

Modo 7

(4.400)

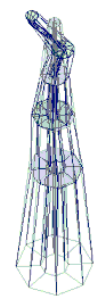

Modo 14

(8.669)

Figura 11. Modos de vibración estimados mediante pruebas de vibración ambiental y sus respectivas frecuencias en $\mathrm{Hz}$ (Ramos et al., 2006) 


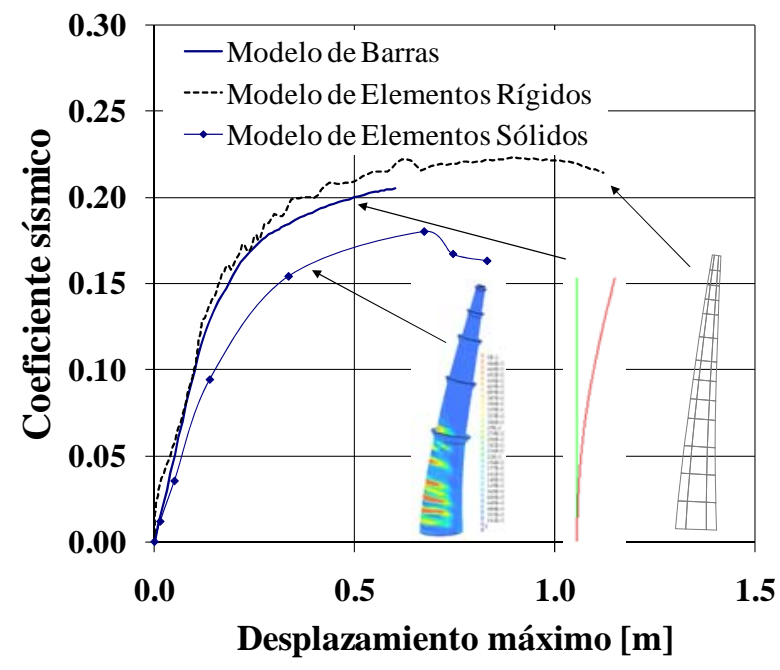

a)

\section{5}

\begin{abstract}
b)
\end{abstract}

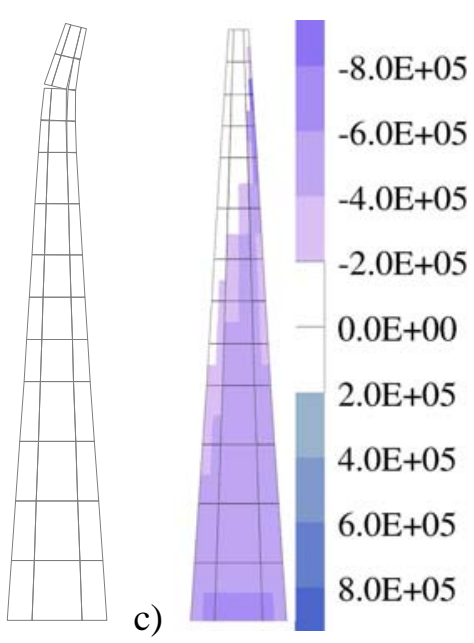

Figura 12. Resultados del análisis no lineal: a) estático (pushover); b) deformada máxima durante el análisis dinámico; c) distribución de esfuerzos axiales en el instante de la máxima deformada en Pa (Peña et al., 2010)

Posteriormente, se realizaron análisis paramétricos con el modelo de barras, los cuales confirmaron que la zona crítica se encontraba en los dos últimos niveles (Fig. 13). Cabe hacer notar que el modelo de barras representa mejor la realidad que el de elementos rígidos, pues entre otras cosas, éste último no toma en cuenta las posibles torsiones de la estructura, ni los diferentes materiales en la sección transversal. Esta limitante se resolvió considerando elementos barra con secciones compuestas.

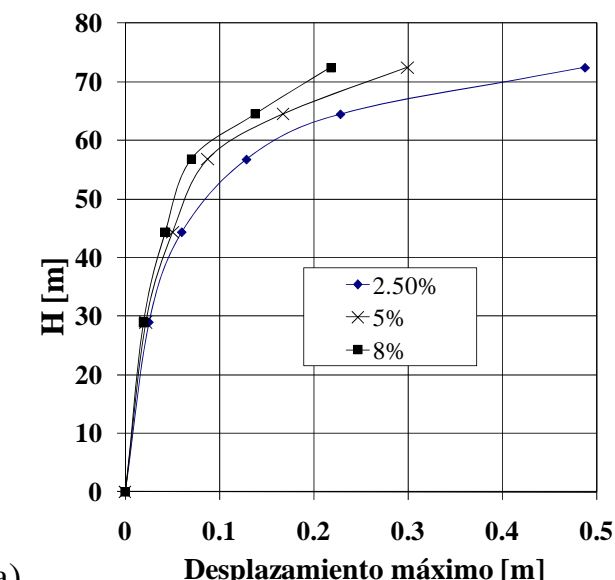

a)

Desplazamiento máximo [m]

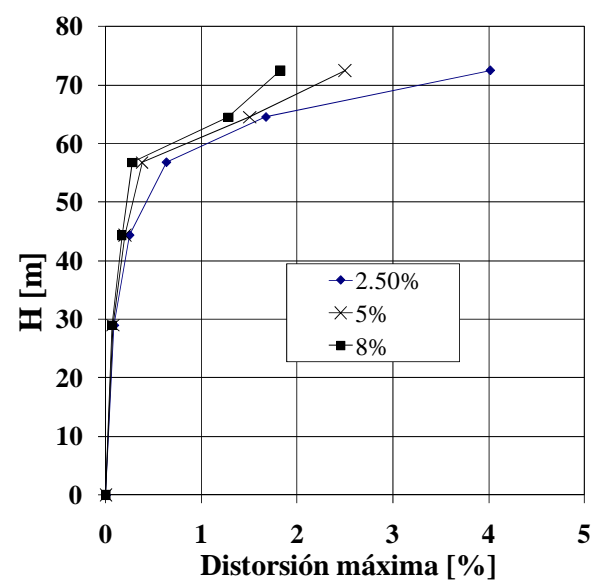

b)

Figura 13. Análisis paramétrico realizado con el modelo de barras, variando el amortiguamiento: a) desplazamiento máximo; b) distorsiones máximas (Peña et al., 2010)

\section{COMENTARIOS FINALES}

La finalidad de una intervención de una estructura histórica debe ser la salvaguarda de sus valores intrínsecos. Por lo tanto, es necesario que el proyecto estructural esté basado en el "entendimiento total" 
del comportamiento estructural del inmueble. De este modo, el diagnóstico y evaluación de la seguridad deben estar basados en un análisis integral de la estructura. Este análisis involucra diversos aspectos, además de un análisis numérico, propiamente dicho, como: análisis de las condiciones pasadas (análisis histórico, análisis de daños e intervenciones), análisis de las condiciones presentes (geometría, materiales), y análisis de las condiciones futuras (posibles cambios y deterioros, riesgo sísmico).

En este contexto, se requiere que el ingeniero tenga un conocimiento global de la estructura, el cual debe forzosamente incluir: "historia clínica de la estructura”, “valoración” y “diagnosis”. De este modo, el ingeniero se debe apoyar en otras disciplinas aparentemente desligadas con la suya, como la Historia y la Arquitectura.

El análisis y modelado numérico se subordina a un proceso de entendimiento más general, y por ende más completo, de la estructura. De este modo, los resultados numéricos de un modelo en particular dejan de ser el objetivo central; pasando a ser sólo un paso intermedio para llegar al objetivo final.

Con base en esto, en este trabajo se propuso una estrategia integral de análisis y modelado numérico la cual se basa en la definición de cinco pasos: adquisición de datos, selección de la herramienta de análisis, proceso de calibración y validación, tipos de análisis y análisis paramétricos. Esta estrategia permite superar las dificultades intrínsecas que se tienen al modelar y analizar este tipo de estructuras.

Así mismo, cabe recordar que, la evaluación de la seguridad estructural de un edificio histórico no debe estar basada únicamente en cálculos numéricos; sino también en las evidencias sobre el comportamiento del edificio ante eventos pasados, es decir, con base en su propia historia.

\section{AGRADECIMIENTOS}

El autor agradece el financiamiento recibido por parte del Programa de Apoyo a Proyectos de Investigación e Innovación Tecnológica (PAPIIT) de la Dirección General de Asuntos del Personal Académico (DGAPA) de la Universidad Nacional Autónoma de México (UNAM), mediante el proyecto PAPIIT IN-105409 “Estrategias para el modelado y análisis de estructuras históricas”.

\section{REFERENCIAS}

Anthoine, A (1995). "Derivation of the in-plane elastic characteristics of masonry through homogenization theory”, International Journal of Solids and Structures, Vol. 32, No. 2, pp. 137163.

Anthoine, A (1997). "Homogenization of periodic masonry: plane stress, generalizaed plane strain or 3D modelling?”, Communications in Numerical Methods in Engineering, Vol. 13, pp. 319-326.

Binda, L, M Luadi y A Saisi A (2007). "Non-destructive testing techniques applied for diagnosis investigation: Syracuse Cathedral in Sicily, Italy”, International Journal of Architectural Heritage, Vol. 1, pp. 380-402.

Brencich, A y S Lagomarsino (1998). “A macroelement dynamic model for masonry shear walls”, Computer Methdos in Structural Masonry- 4, Pande, Middleton y Kralj editors, E\&FN Spon.

Casolo, S y F Peña (2007). "Rigid element model for in-plane dynamics of masonry walls considering hysteretic behaviour and damage”, Earthquake Engineering and Structural Dynamics, Vol. 36, pp. 1029-1048. 
Doglioni, F, A Moretti y V Petrini V (1994). “Le chiese e il terremoto”, Gruppo Nazionale per la difesa dai terremoti, Ed. Lint, Trieste.

Douglas, B M y W H Reid (1982). "Dynamic test and system identification of bridges”, Journal of Structures, ASCE, Vol. 108, pp. 2295-2312.

Eurocódigo 8 (2004). “Design provisions for earthquake resistance of structures. Part 1-1”, EN 1998-1, CEN, Bruselas.

ICOMOS (1964), “Carta internacional sobre la conservación y la restuarción de monumentos y sitios (Carta de Venecia)”, ICOMOS. Disponible en: http://www.icomos.org

ICOMOS (2003), "Principios para el análisis, conservación y restauración de las estructuras del patrimonio arquitectónico”, ICOMOS. Disponible en: http://www.icomos.org

Lemos, J V (2007). "Discrete element modeling of masonry structures”, Journal of Architectural Heritage, Vol. 1, pp. 190-213.

Lourenço, P B (2002). “Computations on historic masonry structures”, Progress in Structural Engineering and Materials, Vol. 4, No. 3, pp. 301-319.

Lourenço, P B (2006). "Recommendations for restoration of ancient buildings and the survival of a masonry chimney”, Construction and Building Materials, Vol. 20, pp. 239-251.

Meli, R y A R Sánchez (1995). "Diagnóstico Estructural”, en: Catedral Metropolitana: corrección geométrica, Informe Técnico, Varios autores, Asociación Amigos de la Catedral Metropolitana de México, A.C., México, pp. 143 - 188.

NTC-S (2004). “Normas Técnicas Complementarias para Diseño por Sismo”, Normas Técnicas Complementarias del Reglamento de Construcciones para el Distrito Federal, Gaceta Oficial del Distrito Federal, Tomo I, No. 103-Bis, México.

Orduña, A y P B Lourenço (2005). “Three-dimensional limit analysis of rigid blocks assemblages. Part I: Torsion failure on frictional interfaces and limit analysis formulation”, International Journal of Solids and Structures, Vol. 42, No. 18-19, pp. 5140-5160.

Orduña, A, G Roeder y F Peña (2007). "Evaluación sísmica de construcciones históricas de mampostería: comparación de tres modelos de análisis”, Revista de Ingeniería Sísmica, SMIS, No. 77, pp. 71-88.

Pegon, P y A Anthoine (1997). "Numerical strategies for solving continuum damage problems with softening: application to the homogenization of masonry”, Computers and Structures, Vol. 64, No. 1-4, pp. 623-642.

Peña, F y S Casolo (2003). “Análisis dinámico de estructuras de mampostería usando un modelo de elementos rígidos: aplicación en el estudio de la seguridad sísmica de una iglesia barroca”, XIV Congreso Nacional de Ingeniería Sísmica, León, Artículo IX-01.

Peña, F y R García Córdova (2004). "Relación de la Ingeniería con la Historia en el proceso de restauración de los edificios históricos”, XIV Congreso Nacional de Ingeniería Estructural, Acapulco, Artículo 55.

Peña, F, S Casolo, y P B Lourenço (2007). "Seismic analysis of masonry monuments by an integrated approach that combines the finite element models with a specific mechanistic model”, IX International Conference on Computational Plasticity, COMPLAS IX, Barcelona, Artículo 235.

Peña, F, P B Lourenço, N Mendes y D Oliveira (2010). "Numerical models for the seismic assessment of an old masonry tower”, Engineering Structures, Vol. 32, pp. 1466-1478. 
Peña, F y J M Meza (2008). "Evaluación de la vulnerabilidad sísmica de torres de campanario de iglesias coloniales en México”, I Congreso Iberoamericano sobre Construcciones Históricas y Estructuras de Mampostería, Bucaramanga, Colombia, pp. 278-286.

RAE (2001). “Diccionario de la lengua española”, Real Academia Española, 22 a Edición, España.

Ramos, L, F Casarín, C Algeri, P B Lourenço y C Modena (2006). "Investigation techniques carried out on the Qutb Minar, New Delhi, India”, Structural Analysis of Historical Constructions, Lourenco, Roca, Modena y Agrawal editores, pp. 633-640. 